\title{
FLUXO DE INFORMAÇÃO EM DESASTRES NATURAIS: os quatro desafios para as ações de resposta
}

\section{Daniel Cardoso}

Mestre em Ciência da Informação pela Universidade Federal de Santa Catarina.

E-mail:

ddnanielcardoso@gmail.com

\section{Adilson Luiz Pinto}

Professor no Programa de PósGraduação em Ciência da Informação da Universidade Federal de Santa Catarina. Doutor em Documentação pela Universidad Carlos III de Madrid

E-mail: adilson.pinto@ufsc.br

\section{RESUMO}

Objetivos: A informação é considerada matéria-prima básica para organizar as ações de resposta a desastres naturais. Sem a informação fluindo de maneira adequada, os gestores e agentes envolvidos nos trabalhos perdem eficiência e não conseguem se organizar da melhor maneira. Este estudo analisou quais são os principais desafios para o fluxo de informação durante os momentos de resposta a desastres naturais. Metodologia: A partir de uma revisão de literatura na Web of Science (1994 até 2014), selecionou-se 17 textos que se enquadravam ao escopo deste estudo. Resultados: 0 estudo destacou quatro desafios que impedem a informação de fluir livremente: Precisão da informação; Volume de informação; Colapso na estrutura de tecnologia e comunicação; Confiança entre as organizações. Conclusão: As principais recomendações propostas neste artigo para facilitar o fluxo de informação são: (i) tratar a informação como matéria-prima fundamental para o planejamento e execução das ações; (ii) criar "filtros" e procedimentos para detectar erros, boatos e impreciões nas informações, priorizando as mais importantes e; (iii) utilizar comunicações via satélite ou rádio amador para evitar quebra no sistema de comunicação.

Palavras-chave: Fluxo de informação; Desastres naturais; Precisão da informação; Volume de informação; Colapso na estrutura de tecnologia e comunicação; Confiança entre as organizações.

\section{INFORMATION FLOW ON NATURAL DISASTERS:} the four challenges for the response actions

\section{ABSTRACT}

Proposal: Information is the basic raw material to organize natural disasters response actions. Without information flowing properly, managers and agents in the work force lose efficiency and cannot manage the 
crisis properly. This situation can result in loss of lives and property. This study analyzed what are the main challenges for the information flow during the response actions to natural disasters. Methodology: From a literature review on Web of Science (1994 until 2014), were selected 17 texts that fit in the scope of this study. Results: The study highlighted four challenges that prevent information from flowing correctly: Accuracy of the information; Volume (amount) of information; Collapse in technology and communication structure; Trust between organizations. Conclusion: The main recommendations in this study to improve information flow are: (i) treat the information as a fundamental raw material for the planning and execution of actions; (ii) create "filters" and procedures to detect mistakes, rumors and low accuracy information, prioritizing the most important and; (iii) use satellite communications and amateur radio to avoid breaks in the communication system.

Keywords: Information flow; Natural disasters; Accuracy of information; Volume of information; Collapse in technology and communication structure; Trust between organizations.

\section{INTRODUÇÃO}

Desastres naturais são considerados importantes problemas tanto pela magnitude quanto por suas consequências socioeconômicas, ambientais e sanitárias nas populações atingidas. Os impactos das áreas afetadas são muitas vezes irreparáveis e causam danos relevantes à saúde das populações, exigindo um esforço integrado de diversos agentes públicos para encaminhar as soluções adequadas em cada caso (SOBRAL et al., 2010).

Desde que as Organizações das Nações Unidas (ONU) iniciaram a série histórica, registrando todos os eventos que ocorrem no mundo, o número de desatres cresce constantemente. Em 1975, primeiro ano da série, a organização registrou 50 ocorrências. Em 2013 (último relatório da organização) o mundo contabilizou em 330 desastres naturais (não incluindo os biológicos), com 21.610 mortes e 96,5 milhões de pessoas atingidas (GUHA-SAPIR; HOYOIS; BELOW, 2013). 
Gráfico 1: número de eventos registrados por países em 2013

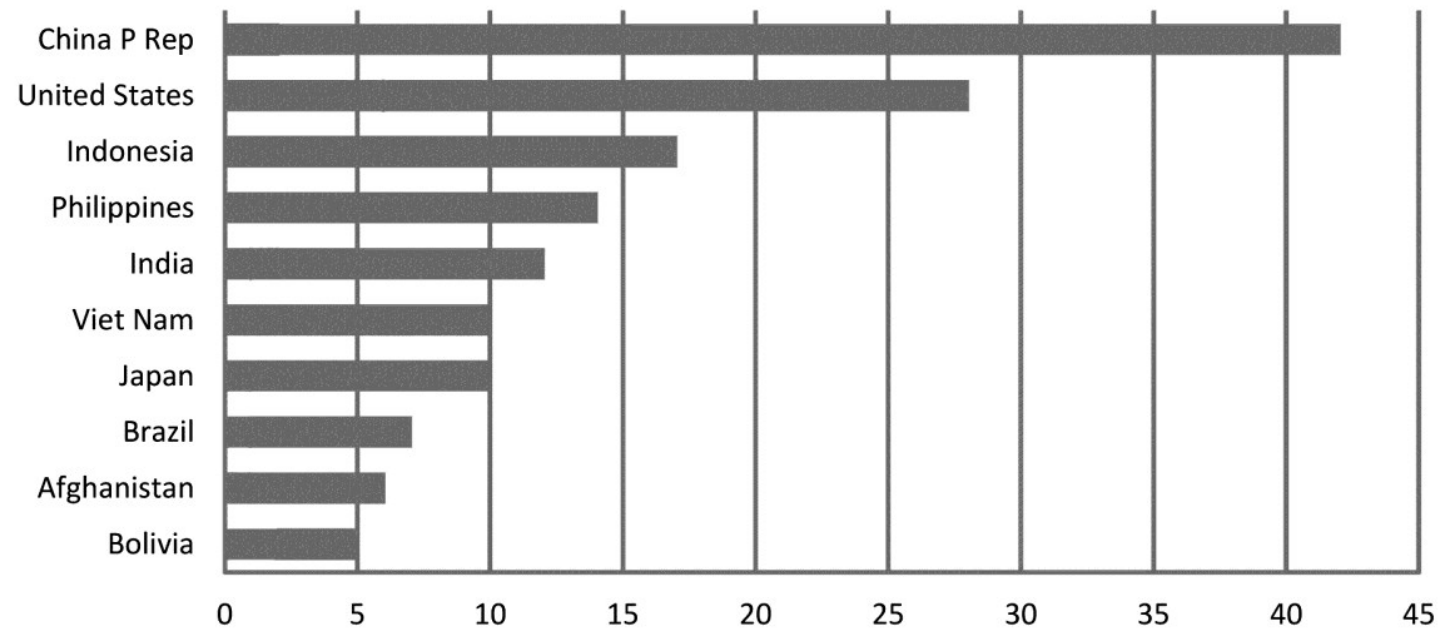

Fonte: (GUHA-SAPIR; HOYOIS; BELOW, 2013, p. 15).

A envergadura e a capilaridade do problema levaram a ONU, em 2005, a elaborar e divulgar o "Hyogo Framework for Action 2005-2015: Building the Resilience of Nations and Communities to Disasters", assinado por todos os paísesmembros das Nações Unidas, o framework tem por objetivo aumentar a resiliência frente aos desastres. 0 documento marcou uma nova fase da $\mathrm{ONU}$, na qual a instituição passou a entender (de maneira mais enfática) que os desastres causam consequências graves para a sobrevivência, dignidade e vida dos indivíduos, em especial dos menos favorecidos financeiramente. No Hyogo Framework for Action são destacadas três ações para reduzir o risco de desastre natural:

a) Desenvolver, atualizar periodicamente e disseminar mapas de risco e informações relativas para os tomadores de decisão, para o público geral e para as comunidades em risco, em um formato apropriado e igualitário;

b) Desenvolver um sistema de indicadores de risco de desastres e vulnerabilidade em escalas nacional e subnacional, que permitam aos tomadores de decisão avaliar o impacto dos desastres na sociedade, economia e meio ambiente e disseminar seus resultados.

c) Armazenar, analisar, sumarizar e disseminar informações estatísticas das ocorrências de desastres, impactos e perdas (ONU, 2005, p. 7). 
Nos três itens supracitados, a palavra informação é ponto central em dois, o que comprova que a gestão de desastres tem na informação uma de suas principais armas para reduzir seus efeitos nocivos. Relevância que também é sustentada pela literatura acadêmica da área de Ciência da Informação.

O livre fluxo da informação e a sua distribuição vem sendo sonhado ao longo de vários momentos históricos e épocas distintas (BARRETO, 2007). 0 fluxo e o compartilhamento da informação promovem ganhos mútuos aos participantes. Isso porque ao obter determinadas informações os atores podem reduzir as incertezas e promover o crescimento entre si (TOMAÉL; MARTELETO, 2006). Sendo que neste artigo consideramos que fluxo de informação é "a sucessão de eventos, de um processo de mediação entre a geração da informação por uma fonte emissora e a aceitação da informação pela entidade receptora" (BARRETO, 1998, p. 122).

Desta forma, este estudo pretende elencar quais são os principais desafios para o fluxo da informação em meio aos trabalhos de resposta aos desastres naturais. A ideia é identificar os quatro maiores gargalos para a troca de informação a partir da revisão da literatura.

\section{DEFINIÇÃO DE DESATRE NATURAL}

Para entender o fluxo de informação em desastres naturais é necessário, antes de tudo, entender o conceito de desastre natural. Na literatura especializada há diferentes definições para conceituar o que é desastre. Todas são muito parecidas entre si e em geral colocam como fato importante o evento ter alterado a rotina de alguma comunidade. A International Federation of Red Cross and Red Crescent Societies - IFRC, por exemplo, entende que desastre natural ocorre quando há repentino, calamitoso evento que interrompe seriamente o funcionamento de uma sociedade ou comunidade e causa perdas humanas, materiais e econômicas que excedem a capacidade de resposta da comunidade ou sociedade. 
A Estratégia Internacional para a Redução de Desastres (UNISDR) elaborou uma definição mais ampla e detalhada, considerando desastre como:

\begin{abstract}
Séria interrupção do funcionamento de uma comunidade ou sociedade que causa perdas humanas e/ou importantes perdas materiais, econômicas ou ambientais; que excedem a capacidade da comunidade ou sociedade afetada de lidar com a situação utilizando seus processos de risco. (UNISDR, 2009, p. 9)
\end{abstract}

Scheuren et al. (2008) é mais objetivo na definição e coloca números palpáveis para delimitar o que é ou não é desastre. Um evento é considerado desastre quando há ocorrência de pelo menos um desses fatores: a) 10 ou mais mortes, b) 100 ou mais pessoas afetadas, c) declaração de estado de emergência ou calamidade pública pelo município, estado ou país.

Mais direto, Araújo (2012) classifica os desastres em dois tipos: os naturais e os humanos (antropogênicos). 0 Centre for Research on the Epidemiology of Disasters (CRED) tem uma metodologia um pouco diferente. Para a organização, há dois tipos de eventos: naturais e tecnológicos. Na categoria desastre natural, há cinco subgrupos. São eles: geofísico, meteorológico, hidrológico, climatológico e biológico.

Com base nos conceitos acima, podemos definir para este artigo que desastre natural é necessariamente algo relativo à ação da Terra impactando na humanidade e que tenha como característica ser calamitoso, atingir populações vulneráveis sem condições de reagir ao evento de forma adequada.

\title{
2.1 As fases de um desastre e o momento da "Resposta"
}

Basicamente, cada desastre possui duas fases: a pré-desastre, subdividida em prevenção, mitigação e risco. A segunda fase é a pós-desastres, subdividida em ações de resposta e ações de recuperação (DEFESA CIVIL DE SANTA CATARINA, 2012). O estudo se concentrou no momento da resposta que é o momento imediatamente antes e imediatamente após o evento em si. Nessa etapa, os esforços se concentram nas necessidades de curto prazo, como conceder 
alimentação, abrigo e socorro às vítimas. A Defesa Civil/SC subdivide a etapa de resposta em três eixos de ação:

- Socorro: atendimento emergencial;

- Assistência: atendimento mediante recursos de logística;

- Restabelecimento: restabelecer serviços essenciais, como abastecimento de água.

Araújo (2012) também descreve as principais ações contempladas dentro da etapa de resposta. Entre elas, podemos citar "Busca e resgate de pessoas afetadas"; "Assistência médica para a população afetada”; “Alojamento temporário, distribuição de alimentos e abrigo à população mais afetada" e "Sistemas de comunicação".

Mas por que concentrar os estudos deste artigo na fase de resposta aos desastres? Justamente pela dificuldade imposta pelo momento. A resposta é uma fase extremamente tensa e que requer execução rápida das ações por causa das vidas e dos bens que estão em risco (NOLTE; BOENIGK, 2011).

Essa fase também exige das organizações e agências envolvidas no trabalho muita agilidade para absorver as informações do que recém-ocorreu e disseminar o conhecimento de maneira eficiente, visando à coordenação das ações de emergência (CHUA; KAYNAK; FOO, 2007).

\section{METODOLOGIA}

Para alcançar o objetivo proposto neste artigo e elencar os principais desafios do fluxo da informação em meio aos trabalhos de resposta em desastres naturais foi realizada uma revisão de literatura. Adotou-se a base de dados Web of Science (de 1994 a 2014) e foram utilizados dois grupos de palavras-chave. 0 primeiro grupo de palavras formado por: "Disaster, networks, disaster response". $\mathrm{Na}$ outra busca adotou-se os termos "Disaster, information management, knowledge management".

Em ambos os casos a busca foi refinada selecionando-se as áreas Public Administration, Social Science e Information Science. Em seguida, selecionou-se os 
artigos mais relevantes de acordo com o número de citações, sendo 25 na primeira busca e 22 na segunda busca.

Com o levantamento pronto, foi realizada uma análise a partir dos resumos dos artigos para verificar aqueles que se adequavam ao escopo. Nesta etapa, foram selecionados 17 artigos (10 na primeira e 7 na segunda busca). Todo o material foi lido na íntegra e do conteúdo extraiu-se os problemas mais recorrentes no fluxo da informação durante desastres naturais. Os problemas destacados estão elencados na tabela abaixo e explicados em detalhes na seção seguinte. A revisão foi realizada em $1^{\circ}$ de junho de 2014.

Quadro 1: Resumo dos quatro desafios para o fluxo de informação

\begin{tabular}{|c|c|c|}
\hline $\begin{array}{l}\text { Desafios para o fluxo de } \\
\text { informação }\end{array}$ & Conceitualização do desafio & Revisão de literatura \\
\hline Precisão da informação & $\begin{array}{l}\text { Durante os trabalhos de respostas aos } \\
\text { desastres naturais, a precisão e a } \\
\text { exatidão das informações são } \\
\text { essenciais para a execução das ações. } \\
\text { Com informações corretas, por } \\
\text { exemplo, o comando pode enviar as } \\
\text { equipes de socorro para os lugares } \\
\text { certos onde pessoas precisam de } \\
\text { ajuda, evitando desperdício de } \\
\text { trabalho e mau uso das equipes. }\end{array}$ & $\begin{array}{l}\text { - COMFORT, 2005, p. 4; } \\
\text { - COMFORT, HAASE, 2006, } \\
\text { p. 4; } \\
\text { - COMFORT, KO, } \\
\text { ZAGORECKI, 2004, p. 305; } \\
\text { - DAY, JUNGLAS, SILVA, } \\
\text { 2009, p. 646; } \\
\text { - LEIDNER, PAN, PAN, 2009, } \\
\text { p. 90; } \\
\text { - YATES; PAQUETTE, 2011, } \\
\text { p.8. }\end{array}$ \\
\hline Volume de informação & $\begin{array}{l}\text { Um dos maiores desafios para os } \\
\text { tomadores de decisão é lidar com o } \\
\text { volume de informação que chega } \\
\text { durante os desastres naturais. Com a } \\
\text { massificação da internet, o uso de } \\
\text { celulares e a atenção da imprensa, é } \\
\text { necessário esforço extra para filtrar } \\
\text { as informações e, em seguida, } \\
\text { priorizar as mais importantes. }\end{array}$ & $\begin{array}{l}\text { - COMFORT, KO, } \\
\text { ZAGORECKI, 2004, p. 309; } \\
\text { - LEIDNER, PAN, PAN, 2009, } \\
\text { p. 89; } \\
\text { - LEIDNER, PAN, PAN, 2012, } \\
\text { p. 32; } \\
\text { - YATES; PAQUETTE, 2011, } \\
\text { p. } 8 .\end{array}$ \\
\hline $\begin{array}{c}\text { Colapso na estrutura de } \\
\text { tecnologia e } \\
\text { comunicação }\end{array}$ & $\begin{array}{l}\text { Desastres naturais costumam afetar a } \\
\text { rede de comunicação com a } \\
\text { derrubada dos sistemas de energia, } \\
\text { internet e telefones (celulares e } \\
\text { convencionais). O colapso impede que } \\
\text { os agentes troquem informações de } \\
\text { maneira ágil e precisa, obrigando-os a } \\
\text { encontrar formas para driblar a falta } \\
\text { de comunicação. }\end{array}$ & $\begin{array}{l}\text { - COMFORT, 2005, p. 3; } \\
\text { - COMFORT, HAASE, 2006, } \\
\text { p. 3; } \\
\text { - DAY, JUNGLAS, SILVA, } \\
\text { 2009, p. 645; } \\
\text { - KAPUCU, ARSLAN, } \\
\text { COLLINS, 2010, p. 223; } \\
\text { - LEIDNER, PAN, PAN, 2009, } \\
\text { p. } 87 .\end{array}$ \\
\hline $\begin{array}{l}\text { Confiança entre as } \\
\text { organizações }\end{array}$ & $\begin{array}{l}\text { Agir em rede (conjunto) com um } \\
\text { objetivo comum e sob estresse requer } \\
\text { um grau elevado de confiança. No } \\
\text { entanto, é comum que rivalidades, } \\
\text { desconfiança e falta de entrosamento } \\
\text { levem o trabalho das instituições ao } \\
\text { fracasso. Por isso, a confiança é }\end{array}$ & $\begin{array}{l}- \text { COMFORT, 2005, p. 10; } \\
-\quad \text { KAPUCU, ARSLAN, } \\
\text { COLLINS, 2010, p. 223; } \\
\text { - } \quad \text { KAPUCU, AUGUSTIN, } \\
\text { GARAYEV, 2009, p. } 300 ; \\
\text { - SIMO, BIES, 2007, p. 127; } \\
\text { - } \text { CHUA, 2007, p. } 1524 ;\end{array}$ \\
\hline
\end{tabular}




\begin{tabular}{|l|l|l|}
\hline & $\begin{array}{l}\text { considerada um dos principais } \\
\text { requisitos para o sucesso nas ações de } \\
\text { resposta aos desastres naturais. }\end{array}$ & $\begin{array}{l}\text { KAPUCU, GARAYEV, } \\
\text { WANG, 2013, p. 107; } \\
\text { - KAPUCU, DEMIROZ, 2011, } \\
\text { p. 551. }\end{array}$ \\
\hline
\end{tabular}

Fonte: Web of Science (2014). Dados da pesquisa.

\section{OS QUATRO DESAFIOS PARA O FLUXO DE INFORMAÇÃO EM DESASTRES}

Nesta seção, serão explanados em detalhes os quatro desafios do fluxo de informação, expostos no quadro 1.

\section{Precisão da Informação}

A revisão de literatura apontou que a precisão da informação é um dos principais desafios encontrados por gestores e agentes que atuam nas respostas aos desastres naturais. Não à toa. A velocidade com que os fatos acontecem, o pouco tempo para apurar as informações e a necessidade de urgência para encaminhar as ações criam um ambiente fértil para erros e imprecisões informacionais.

$\mathrm{Na}$ teoria, o correto é que os agentes recebam informações perfeitas, encontrem as vítimas e entreguem a elas a assistência adequada e de maneira imediata. Porém, na prática, a história é bem diferente. 0 que ocorre é que os agentes de resgaste não sabem exatamente quem precisa do quê, de que tipo de ajuda e em qual localidade as vítimas estão situadas (COMFORT; KO; ZAGORECKI, 2004, p. 299).

A inconsistência nas informações que chegam durante os desastres naturais ocorre porque há múltiplas fontes de informação, que atuam de maneira similar. Essa característica do momento impede que as informações sejam comparadas ou agregadas adequadamente para que se tenha uma definição melhor da situação e dos problemas que surgiram com o desastre (DAY; JUNGLAS; SILVA, 2009). 
A precisão da informação, que é de máxima importância quando se gerencia uma emergência, precisa ser constantemente checada e validada, o que é muito difícil de fazer, devido ao volume de dados que fluem (YATES; PAQUETTE, 2011).

Os autores exemplificam com o caso de 400 imagens que foram lançadas no sistema de informação da Air force Chief of Staff's Crisis Action Team - United States (AFCAT), logo após o terremos do Haiti, em 2010, e que não receberam as tags corretamente. Tal situação dificultou a identificação e, em consequência, a orientação correta para os trabalhos de resposta.

Uma conclusão comum entre os autores que estudam o tema é que a imprecisão e a lacuna de informação tornam-se um fator extremamente limitante de eficiência nas ações de resposta e no trabalho articulado entre diferentes organizações (COMFORT; KO; ZAGORECKI, 2004, p. 305).

Leidner, Pan e Pan (2009) afirmam que uma das maiores dificuldades é fazer com que os membros das equipes, independentemente de onde eles estejam localizados, recebam informações imediatas sobre o que está acontecendo e como responder de maneira adequada.

A imprecisão nas informações pode até mesmo levar ao colapso da rede interorganizacional que atua na resposta aos desastres (COMFORT; HAASE, 2006).

\section{Volume de informação}

O desafio em checar, conferir e enviar informações precisas durante o período de resposta aos desastres naturais é intimamente ligado, e resultado direto de outro desafio comum nesses momentos: o volume de informação. Autores que se debruçam sobre o tema apontam a enorme dificuldade em lidar com a quantidade maciça de informações que chegam a cada segundo, por meio de diferentes plataformas e a partir de uma variada gama de fontes.

Esse volume excessivo dificulta e atrasa a filtragem das informações mais importantes e potencializa o registro de informações incorretas durante momentos de crise (LEIDNER; PAN; PAN, 2012).

Em consequência, o volume excessivo ameaça a eficiência na gestão e na usabilidade das informações recebidas e enviadas (YATES; PAQUETTE, 2011). Por 
isso, os autores afirmam que as organizações precisam garantir processos ou sistemas de controle internos para validar as informações que estão sendo registradas ou compartilhadas.

A dificuldade vem aumentando na proporção da facilidade técnica com que uma informação pode ser transmitida do emissor para o receptor. A quantidade de informação trocada via telefone, telefone sem fio, telefone por satélite, celulares, email, paging, TV, rádio, jornais e internet é gigantesca. A situação deixa ainda mais difícil encontrar meio eficiente de trocar as informações mais relevantes entre as organizações envolvidas nos trabalhos (COMFORT; KO; ZAGORECKI, 2004, p. 309).

Robert Crane Williams e Atiba Phillips escreveram um relatório publicado pela ONU, em 2014, intitulado "Information and communication technologies for disaster risk management in the Caribbean". No relatório, os autores frisam que a turbulência das situações de desastres são propensas a gerar uma superprodução de informação.

Leidner, Pan, Pan (2009) estudaram alguns casos de desastres naturais e também citam o volume de informação como um grande desafio a ser superado. Os autores se basearam nos trabalhos do governo de Cingapura, quando o país enfrentou uma epidemia de Síndrome Respiratória Aguda Severa (SARS), doença que ficou conhecida como Pneumonia Asiática. Nesse caso, o governo enfrentou uma grande dificuldade em disseminar e tratar a informação devido ao número de pacientes e de potenciais indivíduos infectados.

\section{Colapso da infraestrutura de tecnologia e comunicação}

O fluxo de informação tem como principal suporte em situações de emergência e desastres naturais toda a infraestrutura de tecnologia e comunicação existente. 0 papel revolucionário da tecnologia da informação e comunicação (TIC) está na capacidade de conectar grandes redes de indivíduos e organizações entre áreas geográficas distintas e facilitar o rápido fluxo de informação, capital, ideias, pessoas e produtos. As TIC's se tornaram ferramentas essenciais para cooperação e colaboração (WILLIAMS; PHILLIPS, 2014) nos períodos de desastres. 
Vários tipos de TIC's já são utilizados nos momentos de resposta. Desde equipamentos como laptops, servidores de e-mail e servidores de base de dados até tecnologia de informação de segurança de software, como firewall e anti-vírus. Sem essa infraestrutura existente, a informação teria pouco alcance e seria pouco útil (LEIDNER; PAN; PAN, 2009).

A infraestrutura de comunicação precisa suportar as decisões intergovernamentais para capacitar comunidades a responder eficientemente para tal tempestade extensa, rápida e destrutiva (COMFORT; HAASE, 2006). E é por isso que o colapso na infraestrutura se torna um pesadelo real para quem trabalha nesse tipo de operação.

Uma lição importante que precisa ser aprendida é que em desastres irá ocorrer, muito provavelmente, a quebra no sistema de comunicação. Tanto a infraestrutura de telecomunicação quanto a infraestrutura de tecnologia da informação serão rompidas (KAPUCU; ARSLAN; COLLINS, 2010). É algo quase que inevitável, principalmente em desastres muito intensos e geograficamente amplos.

É comum, por exemplo, que um agente de campo se depare com linhas de comunicação interrompidas, estradas inacessíveis, e todo o distrito fechado. Dessa forma, acessar as informações sobre o status do desastre se torna um grande desafio (DAY; JUNGLAS; SILVA, 2009).

O colapso do sistema de operação, especialmente o sistema que conecta a comunicação e os transportes, que permitem a indivíduos e organizações mobilizarem esforços de resposta para auxiliar uma pessoa desamparada ou desabrigada, representa o ponto limiar da falha para a cidade inteira (COMFORT, 2005). Além disso, a falha na infraestrutura determina o nível de performance interorganizacional nas operações (COMFORT; HAASE, 2006).

$\mathrm{Na}$ falta de uma comunicação válida, organizações não funcionam de maneira eficiente debaixo do estresse causado por um desastre. Indivíduos são deixados a tomar suas próprias suposições sobre risco e segurança. Rumores se espalham rapidamente (COMFORT; HAASE, 2006).

Por isso, profissionais de emergência têm praticado/treinado as operações em simulações de queda das linhas telefônicas e de energia, mantendo os geradores funcionando para prover energia e ar-condicionado aos abrigos, além 
das provisões de comida, água e gelo para os residentes (KAPUCU; ARSLAN; COLLINS, 2010).

Quando a estrutura de tecnologia de informação está bem formatada e resiste às intempéries da natureza, há uma chance muito maior de eficiência nas ações de reposta. A infraestrutura existente de tecnologia de informação em Cingapura, por exemplo, provê os fundamentos dos esforços para a gestão de crise. 0 conhecimento de tecnologia de informação entre as entidades rapidamente desenvolveu a capacidade de resposta à crise naquele país (LEIDNER; PAN; PAN, 2009).

No contrário, quando o colapso é total, a situação que já é caótica fica ainda mais crítica. Como no caso citado abaixo, que se refere ao Furacão Katrina que atingiu a cidade de Nova Orleans, nos Estados Unidos.

Talvez o mais sério (problema de planejamento) foi o investimento público inadequado em infraestrutura de comunicação. Quando a enchente atingiu as estações de energia e as torres de telefonia celular, toda a comunicação entrou em colapso. Os rádios se provaram ineficazes e as agências em frequências diferentes não conseguiam se comunicar. Não havia plano B para substituir ou reparar o sistema de comunicação para iniciar as ações chave de resposta. Sem comunicação, a coordenação entre as agências e jurisdições entrou em colapso. (COMFORT, 2005, p. 3)

\section{Confiança entre as organizações da rede de ação}

$\mathrm{Na}$ leitura dos artigos selecionados na revisão de literatura, surgiu um quarto desafio para o fluxo da informação em desastres naturais. Este item não se refere diretamente à forma como a informação é tratada ou disseminada, mas deixa claro que o entrosamento entre os agentes que compõem a rede é preponderante para a qualidade da informação e a eficiência no fluxo da informação.

Para que uma rede de ação seja bem-sucedida, em especial durante momentos de extremo estresse como na gestão de crises, são necessários vários fatores. Entre eles, a confiança mútua. Caso não haja essa confiança, as redes não são benéficas e oferecem pouca ajuda eficiente nas respostas aos desastres naturais (KAPUCU; GARAYEV; WANG, 2013). 
Além de respeito, confiança e interação mútua, as organizações precisam ter a capacidade de colaborar em prol do sucesso das ações (KAPUCU; ARSLAN; COLLINS, 2010). Comfort (2005) lembra que as instituições precisam ter foco claro de trabalho, voltado para a proteção de vidas e das propriedades dos cidadãos em risco. Se não houver essa clareza, todo o sistema tropeça diante de decisões incoerentes e contraditórias.

A desconfiança entre as instituições pode provocar uma espécie de efeito cascata. Se uma entidade não performa seus objetivos de maneira correta e dentro de uma relação de interdependência na rede, alguma outra instituição tende a falhar também e comprometer outras ações importantes (KAPUCU; ARSLAN; COLLINS, 2010).

A natureza de uma rede se desenvolve de acordo com o relacionamento entre os parceiros, sejam organizações ou indivíduos. A qualidade da distribuição dos recursos durante emergências é diretamente proporcional ao funcionamento da rede (KAPUCU; DEMIRO, 2011, p. 551).

Na prática, porém, nem sempre o bom relacionamento é uma característica fácil de ser alcançada. Muitas organizações acumulam anos e anos de rivalidades, falta de confiança e egoísmo, até que consigam ser capazes de participar com sucesso de uma rede de gestão de desastres (KAPUCU; ARSLAN; COLLINS, 2010).

Chua (2007) fez uma revisão de literatura e constatou depoimentos de envolvidos nas ações de respostas reclamando da tensão existente entre as instituições e entre as autoridades que atuam nos trabalhos. É comum que os agentes precisem se equilibrar em meio a uma série de ordens conflitantes, que partem de autoridades diferentes, com constante confronto de opiniões.

Desenvolver a confiança entre agências, apesar das dificuldades, é algo possível. A boa relação precisa ser construída por meio de um aprendizado mútuo e com atividades realizadas em rede, principalmente na fase de prevenção aos desastres. As ações que constroem confiança são complementares à definição das tarefas de cada um, seguida pelo comprometimento com a causa (KAPUCU; AUGUSTIN; GARAYEV, 2009).

As redes precisam ser baseadas na lealdade e na percepção da legitimidade dos trabalhos realizados em parcerias (SIMO; BIES, 2007). Só com o entrosamento 
bem desenvolvido, é que o fluxo de informação poderá circular de maneira eficiente visando o bem comum.

\section{CONCLUSÃO}

A revisão de literatura realizada neste trabalho levou a algumas conclusões. A primeira é a confirmação de que a informação é sim uma matéria-prima essencial para a gestão das ações. Por isso, a informação deve ser encarada como algo estratégico e prioritário no planejamento e execução das ações, seja de prevenção, resposta ou mesmo de reconstução.

Gerenciar a informação, em especial nos momentos de resposta, porém, se mostrou uma missão extremamente árdura, complexa e difícil. Os dois primeiros desafios citados no artigo ("Precisão da informação" e "Volume de informação") são muito ligados entre si e devem ser analisados conjuntamente. Com o uso mais intenso de tecnologias de comunicação, como mensagens sms, whatsapp e e-mail, o volume da informação que chega aos agentes é muito grande. Com o volume alto, cresce também a imprecisão da informação, ou seja, há um risco maior da mensagem estar equivocada, imprecisa ou mesmo de ser mal interpretada, provocando erros na execução das ações ou no planejamento dos trabalhos. Dessa forma, torna-se importante que as entidades da força-tarefa tenham procedimentos para filtrar a informação, priorizando as mais importantes, além de detectar falta de precisão, erros e até mesmo boatos.

O terceiro desafio citado neste artigo, "Colapso na estrutura de tecnologia e comunicação", revela que tais estruturas costumam ser muito suscetíveis aos desastres, principalmente aos de grande magnitude. Por causa disso, os gestores precisam adotar meios de comunicação resistentes ou alterantivos para serem usados nos dias após o desastre. Por exemplo: telefones via satélite ou rádios amadores, que têm mais chances de seguirem funcionando, mesmo após um evento como terremoto ou enchente.

0 último desafio elencado, "Confiança entre as organizações da rede de ação", aponta que é preciso investir muito tempo no relacionamento entre as 
agências e revela que a falta de entrosamento traz consequências graves. A construção desse entrosamento precisa ocorrer no longo prazo e previamente ao evento. A recomendação é que sejam realizadas diversas reuniões, encontros e palestras para definir qual o papel de cada agência tem durante a resposta e que estratégias devem ser adotadas para que não haja sobreposição de funções e atividades. Dessa maneira, cada instituição que atua no momento de resposta saberá o que fazer e a quem reportar, ou mesmo pedir ajuda, favorecendo o fluxo de informação.

\section{REFERÊNCIAS}

ARAÚJO, S. B. Administração de desastres: conceitos e tecnologias. 3. ed. Rio de Janeiro: SYGMA SMS, 2012. Disponível em:

http://www.defesacivil.pr.gov.br/arquivos/File/AdministracaodeDesastres.pdf. Acesso em: 12 jan. 2015.

BARRETO, A. A. Mudança estrutural no fluxo do conhecimento: a comunicação eletrônica. Ciência da Informação, v. 27, n. 2, p. 122-127, 1998. Disponível em: http://www.scielo.br/pdf/ci/v27n2/barreto.pdf. Acesso em: 12 jan. 2015.

BARRETO, A. A. Uma história da Ciência da Informação. In: TOUTAIN, L. M. B. Brandão. (Org.) Para entender a Ciência da Informação. Salvador: EDUFBA, 2007, p. 13-34.

CHUA, A. Y. K. A tale of two hurricane: comparing Katrina and Rita through a knowledge management perspective. Journal of the American Society for Information Science and Technology, v. 58, n. 10, p. 1518-1528, 2007. Disponível em: http://dx.doi.org/10.1002/asi.20640. Acesso em: 15 abr. 2015.

CHUA, A. Y. K; KAYNAK, S.; FOO, S. S. B. An analysis of the delayed response to hurricane katrina through the lens of knowledge management. Journal of the American Society for Information Science and Technology, v. 58, n. 3, p. 391403, 2007. Disponível em: http://dx.doi.org/10.1002/asi.20521. Acesso em: 08 dez. 2014.

COMFORT, K. L. Fragility in disaster response: hurricane Katrina. The Forum, v. 3, n. 3, p. 1-8, 2005. Disponível em: http://dx.doi.org/10.2202/1540-8884.1090. Acesso em: 12 jan. 2015.

COMFORT, K. L.; KO, K.; ZAGORECKI, A. Coordination in rapidly evolving disaster response systems: the role of information. American Behavioral Scientist, v. 48, 
n. 3, p. 295-313, 2004. Disponível em:

http://dx.doi.org/10.1177/0002764204268987. Acesso em: 10 jan. 2015.

COMFORT, L. K.; HAASE, T. W. Communication, coherence, and collective action: the impact of hurricane Katrina on communications infrastructure. Public Works Management \& Policy, v. 11, n. 1, p. 1-16, 2006. Disponível em: http://dx.doi.org/10.1177/1087724X06289052. Acesso em: 22 nov. 2014.

DAY, J. M.; JUNGLAS, I.; SILVA, L. Information flow impediments in disaster relief supply chains. Journal of the Association for Information Systems, v. 10, n. 8, p. 637-660, 2009. Disponível em: http://aisel.aisnet.org/jais/vol10/iss8/1/. Acesso em: 15 jan. 2015.

DEFESA CIVIL DE SANTA CATARINA. Gestão de risco de desastres. 2012

GUHA-SAPIR, D. ; HOYOIS, P.; BELLOW, R. Annual Disaster Statistical Review 2013: The numbers and trends. Bruxelas: CRED, 2013. Disponível em: http://cred.be/sites/default/files/ADSR 2013.pdf. Acesso em: 25 fev. 2015. IFRC - International Federation of Red Cross and Red Crescent Societies. Disponível em: www.ifrc.org/en/what-we-do/disaster-management/aboutdisasters/what-is-a-disaster. Acesso em: 16 dez. 2014.

KAPUCU, N.; ARSLAN, T.; COLLINS, M. L. Examining intergovernmental and interorganizational response to catastrophic disasters: toward a network-centered approach. Administration \& Society, v. 42, n. 2, p. 222-247, 2010. Disponível em: http://dx.doi.org/10.1177/0095399710362517. Acesso 05 fev. 2015.

KAPUCU, N.; AUGUSTIN, M. E.; GARAYEV, V. Interstate partnerships in emergency management: emergency management assistance compact in response to catastrophic disasters. Public Administration Review, v. 69, n. 2, p. 297-313, 2009. Disponível em: http://dx.doi.org/10.1111/j.1540-6210.2008.01975.x. Acesso em: 14 fev. 2015.

KAPUCU, N.; DEMIROZ, F. Measuring performance for collaborative public management using network analysis methods and tools. Public Performance \& Management Review, v. 34, n. 4, p. 549-579, 2011. Disponível em: http://dx.doi.org/10.2753/PMR1530-9576340406. Acesso em: 22 dez. 2014.

KAPUCU, N.; GARAYEV, V.; WANG, X. Sustaining networks in emergency management: a study of counties in the United States. Public Performance \& Management Review, vol. 37, n. 1, p. 104-103, 2013. Disponível em: http://dx.doi.org/10.2753/PMR1530-9576370105. Acesso em: 06 mar. 2015.

LEIDNER, D. E.; PAN, G.; PAN, S. L. Crisis response information networks. Journal of the Association for Information Systems, v. 13, n. 1, p. 31-56, 2012.

Disponível em: http://aisel.aisnet.org/jais/vol13/iss1/1/. Acesso em: 25 fev. 2015. 
LEIDNER, D. E.; PAN, G.; PAN, S. L. The role of IT in crisis response: Lessons from de SARS and Asian Tsunami disasters. Journal of Strategic Information Systems, v. 18, n. 2, p. 80-99, 2009. Disponível em:

http://dx.doi.org/10.1016/i.jsis.2009.05.001. Acesso em: 10 nov. 2014.

NOLTE, I. M.; BOENIGK, S. Public-nonprofit partnership performance in a disaster context: the case of Haiti. Public Administration, v. 89, n. 4, p. 1385-1402, 2011. Disponível em: http://dx.doi.org/10.1111/i.1467-9299.2011.01950.x. Acesso em: 11 dez. 2014.

SCHEUREN, J-M. et al. Annual Disaster Statistical Review - The Numbers and Trends 2008. CRED - Centre for Research on the Epidemiology of Disasters. Brussels: Université Catholique de Louvain, 2008. Disponível em: http://www.cred.be/publication/annual-disaster-statistical-review-numbers-andtrends-2007. Acesso em: 21 de out 2013.

SIMO, G.; BIES, A. L. The role of nonprofits in disaster response: an expanded model of cross-sector collaboration. Public Administration Review, v. 67, n. esp., p. 125142, 2007. Disponível em: http://dx.doi.org/10.1111/j.1540-6210.2007.00821.x. Acesso em: 15 jan. 2015.

SOBRAL, A. et al. Desastres naturais - sistemas de informação e vigilância: uma revisão da literatura. Epidemiologia e Serviços de Saúde, v. 19, n. 4, p. 389-402, 2010. Disponível em: http://dx.doi.org/10.5123/S1679-49742010000400009. Acesso em: 05 jan. 2015.

ONU. Hyogo Framework for Action 2005-2015: Building the resilience of nations and communities to disasters (HFA). 2005. Disponível em: http://www.unisdr.org/2005/wcdr/intergover/official-doc/L-docs/Hyogoframework-for-action-english.pdf. Acesso em: 21out. 2013.

TOMAÉL, M. I; MARTELETO, R. M. Redes Sociais: posições dos atores no fluxo da informação. Encontros Bibli, v.11, n. esp. 1ำ sem, p. 75-91, 2006. Disponível em: http://dx.doi.org/10.5007/1518-2924.2006v11nesp1p75. Acesso em 08 jan. 2015.

UNITED NATIONS INTERNATIONAL STRATEGY FOR DISASTER REDUCTION, p. 9, maio, 2009. Disponível em:

http://www.unisdr.org/files/7817 UNISDRTerminologyEnglish.pdf. Acesso em: 26 maio 2015.

YATES, D.; PAQUETTE, S. Emergency knowledge management and social media technologies: A case study of the 2010 Haitian earthquake. International Journal of Information Management, v. 31, n. 1, p. 6-13, 2011. Disponível em: http://dx.doi.org/10.1016/j.ijinfomgt.2010.10.001. Acesso em 19 de fev. 2015.

WILLIAMS, R. C.; PHILLIPS, A. Information and communication technologies for 
disaster risk management in the Caribeean. Series Studies and Perspectives, ECLAC, ONU, fev., 2014. Disponível em: http://hdl.handle.net/11362/36735. Acesso em 15 mar. 2015.

Recebido em: 26 de junho de 2016 Aceito em: 17 de agosto de 2017 